A. Gogatishvili, Mathematical Institute, Academy of Sciences of the Czech Republic, Žitna 25, 115 67, Prague 1, Czech Republic.

email: gogatish@math.cas.cz

K. Kuliev, Department of Mathematics, University of West Bohemia, Univerzitní 22, 30614, Pilsen, Czech Republic. email: komil@kma.zcu.cz G. Kulieva, Department of Mathematics, University of West Bohemia, Univerzitní 22, 30614, Pilsen, Czech Republic. email: kulieva@kma.zcu.cz

\title{
SOME CONDITIONS CHARACTERIZING THE "REVERSE" HARDY INEQUALITY
}

\begin{abstract}
In this paper, we obtain some characteristic conditions which are equivalent with the validity of the "reverse" Hardy inequality $(-\infty<$ $q \leq p<0)$ and compare these characterizations.
\end{abstract}

\section{Introduction.}

The "reverse" Hardy inequalities

$$
\left(\int_{a}^{b} f^{p}(x) v(x) d x\right)^{\frac{1}{p}} \leq C\left(\int_{a}^{b}\left(\int_{a}^{x} f(t) d t\right)^{q} u(x) d x\right)^{\frac{1}{q}}
$$

and

$$
\left(\int_{a}^{b} f^{p}(x) v(x) d x\right)^{\frac{1}{p}} \leq C\left(\int_{a}^{b}\left(\int_{x}^{b} f(t) d t\right)^{q} u(x) d x\right)^{\frac{1}{q}}
$$

for $f \geq 0$ with given weight functions $u, v$ are completely characterized for $p, q<1$ by P. R. Beesack and H. P. Heinig [1] and for $p, q<0$, and $p, q \in(0,1)$ by D. Prokhorov [6]. A. Kufner and K. Kuliev in [4] also obtained conditions in the case $-\infty<q \leq p<0$.

In [2], the authors have described several scales of conditions for the "classical" Hardy inequality. The aim of this paper is to find similar scales for

Key Words: Inequalities, Hardy's inequality, Weights, Scales of weight characterizations, Hardy operator, Continuity, equivalent integral conditions, comparisons

Mathematical Reviews subject classification: Primary 26D10, 26D15; Secondary 47B38

Received by the editors November 24, 2006

Communicated by: Stefan Schwabik 
the "reverse" Hardy inequality. In next section, we present our main results, namely that an equivalence theorem (see Theorem 2.1) which we will use to give some new scales of weighted characterizations of the "reverse" weighted inequalities (1.1) and (1.2) (see Theorem 2.2).

\section{Scales of Weighted Characterizations.}

We start with an equivalence theorem which we will use in the proof of the main result (Theorem 2.2).

Theorem 2.1. Let $-\infty \leq a<b \leq \infty$. Let $\alpha, \beta, \gamma_{i}(i=1,2)$ be positive and $s_{i}, \theta_{i}(i=1,2)$ be nonnegative numbers such that

$$
s_{i}+\theta_{i}>0 .
$$

(T1) Let $\phi$ be measurable and $\Psi$ be monotone nondecreasing functions, positive a.e. in $(a, b)$, and denote

$$
\Phi(x):=\int_{a}^{x} \phi(t) d t
$$

If we define

$\bar{W}_{1}\left(x ; \alpha, \beta, \gamma_{1}, s_{1}, \theta_{1}\right):=\Phi^{-s_{1}}(x)\left(\int_{a}^{x} \phi(t) \Phi^{\frac{\alpha+s_{1}}{\gamma_{1}}-1}(t) \Psi^{\frac{-\beta+\theta_{1}}{\gamma_{1}}}(t) d t\right)^{\gamma_{1}} \Psi^{-\theta_{1}}(x)$ and

$$
\bar{W}_{2}\left(x ; \alpha, \beta, \gamma_{2}, s_{2}, \theta_{2}\right):=\Phi^{s_{2}}(x)\left(\int_{x}^{b} \phi(t) \Phi^{\frac{\alpha-s_{2}}{\gamma_{2}}-1}(t) \Psi^{\frac{-\beta-\theta_{2}}{\gamma_{2}}}(t) d t\right)^{\gamma_{2}} \Psi^{\theta_{2}}(x),
$$

then the numbers $\bar{W}_{i}\left(\alpha, \beta, \gamma_{i}, s_{i}, \theta_{i}\right)=\sup _{a<x<b} \bar{W}_{i}\left(x ; \alpha, \beta, \gamma_{i}, s_{i}, \theta_{i}\right)(i=$ $1,2)$ are equivalent. Symmetrically,

(T2) Let $\phi$ be measurable and $\Psi$ be monotone nonincreasing functions, positive a.e. in $(a, b)$, and denote

$$
\Phi(x):=\int_{x}^{b} \phi(t) d t
$$

If we define 


$$
\begin{gathered}
\widehat{W}_{1}\left(x ; \alpha, \beta, \gamma_{1}, s_{1}, \theta_{1}\right):=\Phi^{-s_{1}}(x)\left(\int_{x}^{b} \phi(t) \Phi^{\frac{\alpha+s_{1}}{\gamma_{1}}-1}(t) \Psi^{\frac{-\beta+\theta_{1}}{\gamma_{1}}}(t) d t\right)^{\gamma_{1}} \Psi^{-\theta_{1}}(x), \\
\widehat{W}_{2}\left(x ; \alpha, \beta, \gamma_{2}, s_{2}, \theta_{2}\right):=\Phi^{s_{2}}(x)\left(\int_{a}^{x} \phi(t) \Phi^{\frac{\alpha-s_{2}}{\gamma_{2}}-1}(t) \Psi^{\frac{-\beta-\theta_{2}}{\gamma_{2}}}(t) d t\right)^{\gamma_{2}} \Psi^{\theta_{2}}(x)
\end{gathered}
$$

then the numbers $\widehat{W}_{i}\left(\alpha, \beta, \gamma_{i}, s_{i}, \theta_{i}\right)=\sup _{a<x<b} \widehat{W}_{i}\left(x ; \alpha, \beta, \gamma_{i}, s_{i}, \theta_{i}\right)(i=1,2)$ are again equivalent.

In both cases, the equivalence relations can depend on $\alpha, \beta, \gamma_{i}, s_{i}$, and $\theta_{i}(i=$ $1,2)$.

Proof. We show that $\bar{W}_{1}$ and $\bar{W}_{2}$ are equivalent to the number

$$
\bar{W}(\alpha, \beta)=\sup _{a<x<b} \bar{W}(x ; \alpha, \beta)=\sup _{a<x<b} \Phi^{\alpha}(x) \Psi^{-\beta}(x) .
$$

First, we consider the case when $\theta_{i}>0(i=1,2)$. Here we use Theorem 1 in $[2]$, and we put for the functions

$$
f_{i}(t)=\phi(t) \Phi^{\frac{\alpha\left(\theta_{i}-s_{i}\right)}{\gamma_{i} \theta_{i}}-1}(t) \Psi^{-\frac{\alpha+\beta}{\gamma_{i}}}(t), \quad \text { and } \quad G_{i}(x)=\Phi^{\frac{s_{i}}{\theta_{i}}}(x) \Psi(x), \quad i=1,2 .
$$

Then we find

$$
\begin{aligned}
& B_{4}\left(x ; \gamma_{i}, \alpha, \beta\right)=\left(\int_{a}^{x} f_{i}(t) G_{i}^{\frac{\alpha+\beta}{\gamma_{i}}}(t) d t\right)^{\gamma_{i}} G_{i}^{-\beta}(x) \\
& =\left(\int_{a}^{x} \phi(t) \Phi^{\frac{\alpha \theta_{i}+\beta s_{i}}{\gamma_{i} \theta_{i}}-1}(t) d t\right)^{\gamma_{i}} \Phi^{-\frac{\beta s_{i}}{\theta_{i}}}(x) \Psi^{-\beta}(x) \\
& =\left(\frac{\gamma_{i} \theta_{i}}{\left.\alpha \theta_{i}+\beta s_{i}\right)}\right)^{\gamma_{i}} \Phi^{\alpha+\frac{\beta s_{i}}{\theta_{i}}}(x) \Phi^{-\frac{\beta s_{i}}{\theta_{i}}}(x) \Psi^{-\beta}(x), \\
& =C_{i} \Phi^{\alpha}(x) \Psi^{-\beta}(x)=C_{i} \bar{W}(x ; \alpha, \beta), \quad i=1,2, \\
& B_{4}\left(x ; \gamma_{1}, \alpha, \theta_{1}\right)=\left(\int_{a}^{x} f_{1}(t) G_{1}^{\frac{\theta_{1}+\alpha}{\gamma_{1}}}(t) d t\right)^{\gamma_{1}} G_{1}^{-\theta_{1}}(x) \\
& =\left(\int_{a}^{x} \phi(t) \Phi^{\frac{\theta_{1}+\alpha}{\gamma_{1}}-1}(t) \Psi^{\frac{\theta_{1}-\beta}{\gamma_{1}}}(t) d t\right)^{\gamma_{1}} \Phi^{-s_{1}}(x) \Psi^{-\theta_{1}}(x) \\
& =\bar{W}_{1}\left(x ; \alpha, \beta, \gamma_{1}, s_{1}, \theta_{1}\right)
\end{aligned}
$$

and

$$
\begin{aligned}
B_{2}\left(x ; \gamma_{2}, \alpha, \theta_{2}\right) & =\left(\int_{x}^{b} f_{2}(t) G_{2}^{\frac{-\theta_{2}+\alpha}{\gamma_{2}}}(t) d t\right)^{\gamma_{2}} G_{2}^{\theta_{2}}(x) \\
& =\bar{W}_{2}\left(x ; \alpha, \beta, \gamma_{2}, s_{2}, \theta_{2}\right) .
\end{aligned}
$$


Denote

$$
B_{4}\left(\gamma_{i}, \alpha, \beta\right):=\sup _{a<x<b} B_{4}\left(x ; \gamma_{i}, \alpha, \beta\right)
$$

and

$$
B_{6-2 i}\left(\gamma_{i}, \alpha, \theta_{i}\right):=\sup _{a<x<b} B_{6-2 i}\left(x ; \gamma_{i}, \alpha, \theta_{i}\right) \quad i=1,2 .
$$

According to the Theorem 1 in [2], we have that

$$
B_{4}\left(\gamma_{1}, \alpha, \beta\right) \approx B_{4}\left(\gamma_{1}, \alpha, \theta_{1}\right) \quad \text { and } \quad B_{4}\left(\gamma_{2}, \alpha, \beta\right) \approx B_{2}\left(\gamma_{2}, \alpha, \theta_{2}\right) \text {. }
$$

Therefore, we obtain

$$
\bar{W}_{1}\left(\alpha, \beta, \gamma_{1}, s_{1}, \theta_{1}\right) \approx B_{4}\left(\gamma_{1}, \alpha, \beta\right)=C_{1} \bar{W}(\alpha, \beta)
$$

and

$$
\bar{W}_{2}\left(x ; \alpha, \beta, \gamma_{2}, s_{2}, \theta_{2}\right) \approx B_{4}\left(\gamma_{2}, \alpha, \beta\right)=C_{2} \bar{W}(\alpha, \beta)
$$

Consequently, we get the equivalence of $\bar{W}_{1}$ and $\bar{W}_{2}$. Let $\theta_{i}=0$. Then $s_{i}>0(i=1,2)$ since $(2.1)$, and the expressions $\bar{W}_{1}$ and $\bar{W}_{2}$ take the forms

$$
\bar{W}_{1}\left(x ; \alpha, \beta, \gamma_{1}, s_{1}, 0\right)=\Phi^{-s_{1}}(x)\left(\int_{a}^{x} \phi(t) \Phi^{\frac{\alpha+s_{1}}{\gamma_{1}}-1}(t) \Psi^{-\frac{\beta}{\gamma_{1}}}(t) d t\right)^{\gamma_{1}}
$$

and

$$
\bar{W}_{2}\left(x ; \alpha, \beta, \gamma_{2}, s_{2}, 0\right)=\Phi^{s_{2}}(x)\left(\int_{x}^{b} \phi(t) \Phi^{\frac{\alpha-s_{2}}{\gamma_{2}}-1}(t) \Psi^{-\frac{\beta}{\gamma_{2}}}(t) d t\right)^{\gamma_{2}} .
$$

Then by the monotonicity of $\Psi$, we get

$$
\begin{aligned}
\bar{W}_{1}\left(x ; \alpha, \beta, \gamma_{1}, s_{1}, 0\right) & \geq \Phi^{-s_{1}}(x)\left(\int_{a}^{x} \phi(t) \Phi^{\frac{\alpha+s_{1}}{\gamma_{1}}-1}(t) d t\right)^{\gamma_{1}} \Psi^{-\beta}(x) \\
& =\left(\frac{\gamma_{1}}{\alpha+s_{1}}\right)^{\gamma_{1}} \bar{W}(x ; \alpha, \beta),
\end{aligned}
$$

and

$$
\begin{aligned}
\bar{W}_{2}\left(x ; \alpha, \beta, \gamma_{2}, s_{2}, 0\right) & \geq \Phi^{s_{2}}(x)\left(\int_{x}^{b} \phi(t) \Phi^{\frac{\alpha-s_{2}}{\gamma_{2}}-1}(t) \Psi^{-\frac{\beta+s_{2}}{\gamma_{2}}}(t) d t\right)^{\gamma_{2}} \Psi^{s_{2}}(x) \\
& =\bar{W}_{2}\left(x ; \alpha, \beta, \gamma_{2}, s_{2}, s_{2}\right) .
\end{aligned}
$$

From these and from the above (namely, the case $\theta_{2}>0$ ), we get

$$
\bar{W}_{i}\left(\alpha, \beta, \gamma_{i}, s_{i}, 0\right) \geq C_{i} \bar{W}(\alpha, \beta) \quad i=1,2 .
$$


The inverse inequality also holds since

$\bar{W}_{1}\left(x ; \alpha, \beta, \gamma_{1}, s_{1}, 0\right) \leq \Phi^{-s_{1}}(x)\left(\int_{a}^{x} \phi(t) \Phi^{\frac{s_{1}}{\gamma_{1}}-1}(t) d t\right)^{\gamma_{1}} \bar{W}(\alpha, \beta)=\left(\frac{\gamma_{1}}{s_{1}}\right)^{\gamma_{1}} \bar{W}(\alpha, \beta)$

and

$\bar{W}_{2}\left(x ; \alpha, \beta, \gamma_{2}, s_{2}, 0\right) \leq \Phi^{s_{2}}(x)\left(\int_{x}^{b} \phi(t) \Phi^{-\frac{s_{2}}{\gamma_{2}}-1}(t) d t\right)^{\gamma_{2}} \bar{W}(\alpha, \beta) \leq\left(\frac{\gamma_{2}}{s_{2}}\right)^{\gamma_{2}} \bar{W}(\alpha, \beta)$.

Thus, we have proved part (T1) of the theorem. Part (T2) can be proved analogously as part $(T 1)$, namely, let $\theta_{i}>0(i=1,2)$, and we choose for the functions

$$
G_{i}(x)=\Phi^{-\frac{s_{i}}{\theta_{i}}}(x) \Psi^{-1}(x) \quad \text { and } \quad f_{i}(x)=\phi(x) \Phi^{\frac{\alpha}{\gamma_{i}}+\frac{\beta s_{i}}{\gamma_{i} \theta_{i}}-1}(x), \quad i=1,2 .
$$

Then we find that

$$
\begin{aligned}
B_{1}\left(x ; \gamma_{i}, \beta\right) & =\left(\int_{x}^{b} f_{i}(t) d t\right)^{\gamma_{i}} G_{i}^{\beta}(x)=C_{i} \widehat{W}(x ; \alpha, \beta), \quad i=1,2, \\
B_{2}\left(x ; \gamma_{1}, \beta, \theta_{1}\right) & =G_{1}^{\theta_{1}}(x)\left(\int_{x}^{b} f_{1}(t) G_{1}^{\frac{\beta-\theta_{1}}{\gamma_{1}}}(t) d t\right)^{\gamma_{1}}=\widehat{W}_{1}\left(x ; \alpha, \beta, \gamma_{1}, s_{1}, \theta_{1}\right)
\end{aligned}
$$

and

$$
B_{4}\left(x ; \gamma_{2}, \beta, \theta_{2}\right)=G_{2}^{-\theta_{2}}(x)\left(\int_{a}^{x} f_{2}(t) G_{2}^{\frac{\beta+\theta_{2}}{\gamma_{2}}}(t) d t\right)^{\gamma_{2}}=\widehat{W}_{2}\left(x ; \alpha, \beta, \gamma_{2}, s_{2}, \theta_{2}\right),
$$

where $\widehat{W}(x ; \alpha, \beta)=\Phi^{\alpha}(x) \Psi^{-\beta}(x)$ and $B_{i}(i=1,2,4)$ were defined in Theorem 1 in [2]. The proof of $\theta_{i}=0(i=1,2)$ follows by the same ideas as the proof of the case $\theta_{i}=0$ in part (T1). The proof is complete.

Theorem 2.1 is an analogue and little extension of the equivalence theorem in [2] (Theorem 1) and allows to give equivalent characterizations of the "reverse" Hardy inequality (1.1) and (1.2).

Theorem 2.2. Let $-\infty<q \leq p<0$ and $p^{\prime}=\frac{p}{p-1}, q^{\prime}=\frac{q}{q-1}$. Let $\lambda_{i}, \mu_{i}$, and $\nu_{i}(i=1,2)$ be real parameters such that $\nu_{i}>0$ and $\lambda_{i}, \mu_{i} \in(-\infty, 1]$, and

$$
\lambda_{i}+\mu_{i}<2 \text {. }
$$

(T'1) Denote

$$
U(x):=\int_{a}^{x} u(t) d t, \quad V(x):=\int_{a}^{x} v^{1-p^{\prime}}(t) d t
$$


and suppose

$$
V(x)<\infty \quad \text { and } \quad U(x)<\infty \quad \text { for } \quad x \in(a, b) .
$$

Define

$$
\begin{aligned}
& \bar{A}_{1}\left(x ; \lambda_{1}, \mu_{1}, \nu_{1}\right):=U^{\frac{\lambda_{1}-1}{q^{\prime}}}(x)\left(\int_{a}^{x} u(t) U^{\frac{2-q^{\prime}-\lambda_{1}}{q^{\prime} \nu_{1}}-1}(t) V^{\frac{\mu_{1}-p}{p \nu_{1}}}(t) d t\right)^{\nu_{1}} V^{\frac{1-\mu_{1}}{p}}(x), \\
& \bar{A}_{2}\left(x ; \lambda_{2}, \mu_{2}, \nu_{2}\right):=U^{\frac{1-\lambda_{2}}{q^{\prime}}}(x)\left(\int_{x}^{b} u(t) U^{\frac{\lambda_{2}-q^{\prime}}{q^{\prime} \nu_{2}}-1}(t) V^{\frac{2-p-\mu_{2}}{p \nu_{2}}}(t) d t\right)^{\nu_{2}} V^{\frac{\mu_{2}-1}{p}}(x) .
\end{aligned}
$$

Then any of the weighted inequality (1.1) holds for all measurable functions $f \geq 0$ if and only if any of the quantities

$$
\bar{A}_{i}\left(\lambda_{i}, \mu_{i}, \nu_{i}\right)=\sup _{a<x<b} \bar{A}_{i}\left(x ; \lambda_{i}, \mu_{i}, \nu_{i}\right)
$$

is finite. Moreover, for the best constant $C$ in (1.1), we have $C \approx \bar{A}_{i}, i=$ 1,2. Symmetrically,

(T'2) Denote

$$
U(x):=\int_{x}^{b} u(t) d t, \quad V(x):=\int_{x}^{b} v^{1-p^{\prime}}(t) d t,
$$

and suppose

$$
V(x)<\infty \text { and } U(x)<\infty \text { for } x \in(a, b) .
$$

Define

$$
\begin{aligned}
& \widehat{A}_{1}\left(x ; \lambda_{1}, \mu_{1}, \nu_{1}\right):=U^{\frac{\lambda_{1}-1}{q^{\prime}}}(x)\left(\int_{x}^{b} u(t) U^{\frac{2-q^{\prime}-\lambda_{1}}{q^{\prime} \nu_{1}}-1}(t) V^{\frac{\mu_{1}-p}{p \nu_{1}}}(t) d t\right)^{\nu_{1}} V^{\frac{1-\mu_{1}}{p}}(x), \\
& \widehat{A}_{2}\left(x ; \lambda_{2}, \mu_{2}, \nu_{2}\right):=U^{\frac{1-\lambda_{2}}{q^{\prime}}}(x)\left(\int_{a}^{x} u(t) U^{\frac{\lambda_{2}-q^{\prime}}{q^{\prime} \nu_{2}}-1}(t) V^{\frac{2-p-\mu_{2}}{p \nu_{2}}}(t) d t\right)^{\nu_{2}} V^{\frac{\mu_{2}-1}{p}}(x) .
\end{aligned}
$$

Then any of the weighted inequality (1.2) holds for all measurable functions $f \geq 0$ if and only if any of the quantities

$$
\widehat{A}_{i}\left(\lambda_{i}, \mu_{i}, \nu_{i}\right)=\sup _{a<x<b} \widehat{A}_{i}\left(x ; \lambda_{i}, \mu_{i}, \nu_{i}\right)
$$

is finite. Moreover, for the best constant $C$ in (1.2), we have $C \approx \widehat{A}_{i}, i=$ 1,2 . 
Proof. In Theorem 2.1, we put $\alpha=-\frac{1}{q}, \beta=\frac{1}{p^{\prime}}, \gamma_{i}=\nu_{i}, s_{i}=\frac{1-\lambda_{i}}{q^{\prime}}$, and $\theta_{i}=\frac{\mu_{i}-1}{p}(i=1,2)$, and the condition (2.1) was satisfied because of (2.2).

$\left(T^{\prime} 1\right)$ We choose for the functions $\phi(x)=u(x)$, and $\Psi(x)=V(x)$. Then

$$
\Phi(x)=U(x)=\int_{a}^{x} u(t) d t .
$$

Then the assertion follows from the fact that

$$
\begin{aligned}
\bar{A} & =\sup _{a<x<b} \bar{W}\left(x ;-\frac{1}{q}, \frac{1}{p^{\prime}}\right), \\
\bar{A}_{1}\left(\lambda_{1}, \mu_{1}, \nu_{1}\right) & =\sup _{a<x<b} \bar{W}_{1}\left(x ;-\frac{1}{q}, \frac{1}{p^{\prime}}, \nu_{1}, \frac{1-\lambda_{1}}{q^{\prime}}, \frac{\mu_{1}-1}{p}\right), \\
\bar{A}_{2}\left(\lambda_{2}, \mu_{2}, \nu_{2}\right) & =\sup _{a<x<b} \bar{W}_{2}\left(x ;-\frac{1}{q}, \frac{1}{p^{\prime}}, \nu_{2}, \frac{1-\lambda_{2}}{q^{\prime}}, \frac{\mu_{2}-1}{p}\right)
\end{aligned}
$$

are all equivalent to $\bar{A}$ according to Theorem 2.1, and the finiteness of $\bar{A}$ is necessary and sufficient for the inequality (1.1) to hold. Moreover, since for the least constant $C$ in (1.1), we have $C \approx \bar{A}$, it is clear that $C \approx \bar{A}_{i}$.

$\left(T^{\prime} 2\right)$ We choose for the functions $\phi(x)=u(x)$ and $\Psi(x)=V(x)$. Then

$$
\Phi(x)=U(x)=\int_{x}^{b} u(t) d t .
$$

Then the assertion follows from the fact that

$$
\begin{aligned}
\widehat{A} & =\sup _{a<x<b} \widehat{W}\left(x ;-\frac{1}{q}, \frac{1}{p^{\prime}}\right), \\
\widehat{A}_{1}\left(\lambda_{1}, \mu_{1}, \nu_{1}\right) & =\sup _{a<x<b} \widehat{W}_{1}\left(x ;-\frac{1}{q}, \frac{1}{p^{\prime}}, \nu_{1}, \frac{1-\lambda_{1}}{q^{\prime}}, \frac{\mu_{1}-1}{p}\right), \\
\widehat{A}_{2}\left(\lambda_{2}, \mu_{2}, \nu_{2}\right) & =\sup _{a<x<b} \widehat{W}_{2}\left(x ;-\frac{1}{q}, \frac{1}{p^{\prime}}, \nu_{2}, \frac{1-\lambda_{2}}{q^{\prime}}, \frac{\mu_{2}-1}{p}\right)
\end{aligned}
$$

are all equivalent to $\widehat{A}$ according to Theorem 2.1, and the finiteness of $\widehat{A}$ is necessary and sufficient for the inequality (1.2) to hold. Moreover, since for the least constant $C$ in (1.2), we have $C \approx \widehat{A}$, it is clear that $C \approx \widehat{A}_{i}$. The proof is complete.

Remark 2.3. (i) In Prokhorov [6], it is shown that the validity of the inequalities

$$
\left(\int_{a}^{b} g^{p}(x) d x\right)^{\frac{1}{p}} \leq C\left(\int_{a}^{b}\left(\int_{a}^{x} g(t) w(t) d t\right)^{q} u(x) d x\right)^{\frac{1}{q}}
$$


and

$$
\left(\int_{a}^{b} g^{p}(x) d x\right)^{\frac{1}{p}} \leq C\left(\int_{a}^{b}\left(\int_{x}^{b} g(t) w(t) d t\right)^{q} u(x) d x\right)^{\frac{1}{q}}
$$

are equivalent with the finiteness of the expressions

$$
A_{P}:=\sup _{a<x<b}\left(\int_{a}^{x} u(t) d t\right)^{-\frac{1}{q}}\left(\int_{a}^{x} w^{p^{\prime}}(t) d t\right)^{-\frac{1}{p^{\prime}}}
$$

and

$$
A_{P}^{*}:=\sup _{a<x<b}\left(\int_{x}^{b} u(t) d t\right)^{-\frac{1}{q}}\left(\int_{x}^{b} w^{p^{\prime}}(t) d t\right)^{-\frac{1}{p^{\prime}}}
$$

respectively. Since $A_{P}$ and $A_{P}^{*}$ coincide with the $\bar{A}$ and $\widehat{A}$ from (2.5) and (2.6), respectively, Prokhorov's results follow from Theorem 2.2. Inequalities (2.7) and (2.8) can be obtained from (1.1) and (1.2) by replacing the function $f(t)$ by $g(t) v^{-\frac{1}{p}}(t)$.

(ii) Similarly, the results of A. Kufner \& K. Kuliev [4] also follow from Theorem 2.2 since their conditions of the validity of (1.1) and (1.2) read:

$$
A_{K}(s):=\sup _{a<x<b}\left(\int_{a}^{x} u(t) V^{\frac{p-s}{p} q}(t) d t\right)^{-\frac{1}{q}} V^{\frac{1-s}{p} q}(x)<\infty
$$

and

$$
A_{K}^{*}(s):=\sup _{a<x<b}\left(\int_{x}^{b} u(t) V^{\frac{p-s}{p} q}(t) d t\right)^{-\frac{1}{q}} V^{\frac{1-s}{p} q}(x)<\infty,
$$

and it is

$$
\begin{aligned}
& A_{K}=\bar{A}_{1}\left(1, s,-\frac{1}{q}\right), \\
& A_{K}^{*}=\widehat{A}_{1}\left(1, s,-\frac{1}{q}\right) .
\end{aligned}
$$

Acknowledgement. The research of the first author was partly supported by the grant 201/05/2033 of the Grant Agency of the Czech Republic and by the Institutional Research plan no. AV0Z10190503 of the Academy of Sciences of the Czech Republic (ASCR).

\section{References}

[1] P. R. Beesack and H. P. Heinig, Hardy's inequalities with indices less than 1, Proc. Amer. Math. Soc., 83(3) (1981), 532-536. 
[2] A. Gogatishvili, A. Kufner, L. E. Persson, A. Wedestig, An equivalence theorem for integral conditions related to Hardy's inequality, Real Anal. Exchange, 29(2) (2003/04), 867-880.

[3] A. Kufner, L. E. Persson, A. Wedestig, A study of some constant characterizing the weighted Hardy inequality, Banach Center Publ., 64 (2004), $135-146$.

[4] A. Kufner and K. Kuliev, The Hardy inequality with "negative powers", Adv. Alg. Anal., 1(3) (2006), 219-228.

[5] B. Opic and A. Kufner, Hardy-type inequalities, Pitman Research Notes in Mathematics, 219, Longman Scientific \& Technical, Harlow, 1990.

[6] D. V. Prokhorov, Weighted Hardy's inequalities for negative indices, Publ. Mat., 48 (2004), 423-443. 
